\title{
Effect of Locked-Nucleic Acid on a Biologically Active G-Quadruplex. A Structure-Activity Relationship of the Thrombin Aptamer
}

\author{
Laura Bonifacio ${ }^{1}$, Frank C. Church ${ }^{2}$ and Michael B. Jarstfer ${ }^{1, *}$
}

1 Division of Medicinal Chemistry and Natural Products, School of Pharmacy, University of North Carolina at Chapel Hill Chapel Hill, North Carolina 27599, USA

2 Departments of Pathology and Laboratory Medicine, Pharmacology and Medicine, Carolina Cardiovascular Biology Center, School of Medicine, University of North Carolina at Chapel Hill Chapel Hill, North Carolina 27599, USA

* Author to whom correspondence should be addressed; School of Pharmacy, Division of Medicinal Chemistry and Natural Products, CB \# 7360, University of North Carolina at Chapel Hill, Chapel Hill, NC 27599-7360, USA. Tel.: 919-966-6422; Fax: 919-966-0204; E-mail: jarstfer@unc.edu

Received: 23 January 2008; in revised form: 17 March 2007 / Accepted: 18 March 2008 / Published: 24 March 2008

\begin{abstract}
Here we tested the ability to augment the biological activity of the thrombin aptamer, d(GGTTGGTGTGGTTGG), by using locked nucleic acid (LNA) to influence its G-quadruplex structure. Compared to un-substituted control aptamer, LNA-containing aptamers displayed varying degrees of thrombin inhibition. Aptamers with LNA substituted in either positions G5, T7, or G8 showed decreased thrombin inhibition, whereas LNA at position G2 displayed activity comparable to un-substituted control aptamer. Interestingly, the thermal stability of the substituted aptamers does not correlate to activity - the more stable aptamers with LNA in position G5, T7, or G8 showed the least thrombin inhibition, while a less stable aptamer with LNA at G2 was as active as the un-substituted aptamer. These results suggest that LNA substitution at sites G5, T7, and G8 directly perturbs aptamer-thrombin affinity. This further implies that for the thrombin aptamer, activity is not dictated solely by the stability of the G-quadruplex structure, but by specific interactions between the central TGT loop and thrombin and that LNA can be tolerated in a biologically active nucleic acid structure albeit in a position dependent fashion.
\end{abstract}

Keywords: aptamer, G-quadruplex, thrombin, locked nucleic acid (LNA) 


\section{Introduction}

Nucleic acids that fold into G-quadruplexes have attracted significant attention because of their potential therapeutic applications. G-quadruplexes with anti-HIV [1], anti-proliferative [2], and anti-thrombin [3,4] activities have recently been identified. In 1993, Griffin et al. [3,4] used SELEX to identify a 15 nucleotide DNA G-quadruplex with the sequence d(GGTTGGTGTGGTTGG) that demonstrates anti-thrombin activity. The thrombin aptamer offers several potential clinical advantages over more traditional drugs for use in anticoagulation. For example, the short half-life of the thrombin aptamer makes it rapidly reversible. Further, this targeted oligonucleotide displays high specificity, which decreases the potential for untoward effects. The interaction between thrombin and the DNA thrombin aptamer has been characterized by x-ray crystallography and NMR, and binding was shown to occur at exosite 1 (the anion binding exosite) [4,5]. This binding mechanism confers yet another advantage to the thrombin aptamer; because it binds at thrombin exosite-1 (rather than the active site) the thrombin aptamer is able to inhibit both free and clot-bound thrombin [6]. Structurally, the thrombin aptamer folds into an intramolecular, chair-like G-quadruplex with two stacked G-tetrads and three flanking loops (Figure 1) [7]. Despite this seemingly well-defined structure, several unique folding topologies of the thrombin aptamer are possible [8]. To what extent these contribute to the biological activity of the thrombin aptamer is not known and can only be accurately addressed if specific folding topologies can be produced.

Figure 1. Structure of thrombin aptamer (adapted from Kelly et al [8]). Residues G2, T4, G5, T7, and G8 are individually substituted with LNA analogs in the following experiments.

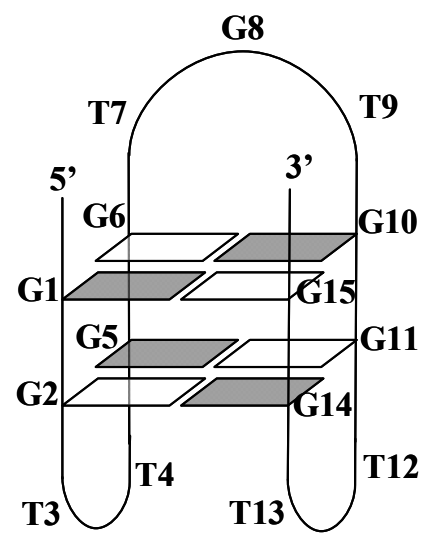

Recently, we found that incorporating a single locked nucleic acid (LNA) residue into a DNA G-quadruplex can influence its structure [9]. LNA are 2'-O-4'-C-methylene-linked ribonucleotide nucleic acid analogues that bind with increased affinity to DNA and RNA oligomers due to a forced 3 'endo conformation of the glycosidic residue [10,11]. LNA-containing oligonucleotides have been utilized for various applications including defining the HIV-1 PPT recognition by HIV-1 RT [12], as probes in developing real-time PCR protocol for genotype analysis [13], and to increase efficiency in targeting antisense oligonucleotides with various ribozymes [14]. In general, LNA has been most extensively explored to increase the affinity of oligonucleotides for DNA and RNA targets [15]. The bicyclic structure of LNA forces the sugar to be in the C3'-endo conformation, and nucleotides with a C3'-endo conformation prefer the glycosidic bond to be in the anti configuration. Because 
G-quadruplexes have very specific requirements for the sugar pucker and glycosidic bond configuration [16], we reasoned that a strategically placed LNA would influence the G-quadruplex folding topology of the thrombin aptamer potentially affecting its activity. As we were completing our work, the effect of extensive LNA substitution on the structure of the thrombin aptamer was reported [17], but the biological effect of only a single LNA at the terminus of the thrombin aptamer was reported [18]. Here, we examined the effect of LNA substitutions at several positions in the thrombin aptamer on its activity.

\section{Results and Discussion}

\section{Biophysical Characterization of Thrombin Aptamers}

In a previous communication, we reported the finding that LNAs can affect the folding topology of G-quadruplex DNA [9]. This prompted us to examine the ability of LNAs to influence the activity of a biologically active G-quadruplex. We examined the G-quadruplex forming thrombin aptamer, d(GGTTGGTGTGGTTGG), containing single LNA substitutions in several positions, which are indicated in Table 1. We first determined the effect of the LNAs on the folding topology of the thrombin aptamer. Using CD spectroscopy, we found that the thrombin aptamer and each LNAcontaining derivative produced a strong, positive CD absorbance at $295 \mathrm{~nm}$ with a minimum at 260 $\mathrm{nm}$, indicative of an anti-parallel quadruplex (Figure 2).

Figure 2. CD spectra of thrombin aptamer and LNA-containing thrombin aptamers. CD spectra were obtained using $5 \mu \mathrm{M}$ oligonuceotide in $10 \mathrm{mM}$ Tris buffer, $\mathrm{pH} 7.5$, containing $1 \mathrm{mM}$ EDTA and $50 \mathrm{mM} \mathrm{KC1}$. ATA is a scrambled aptamer (see Experimental Procedures).

A.

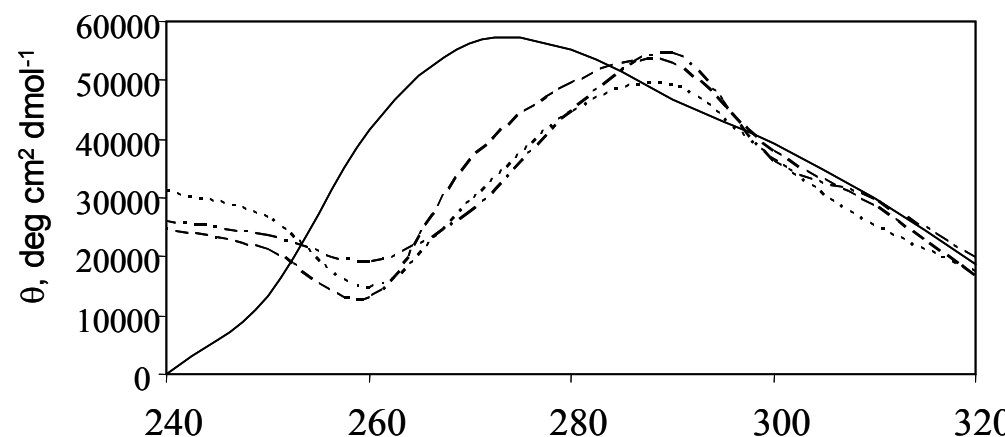

- SATA

$-\cdot \cdot \cdot$ ATA

…... LNA-G2

---- LNA-T4

B.

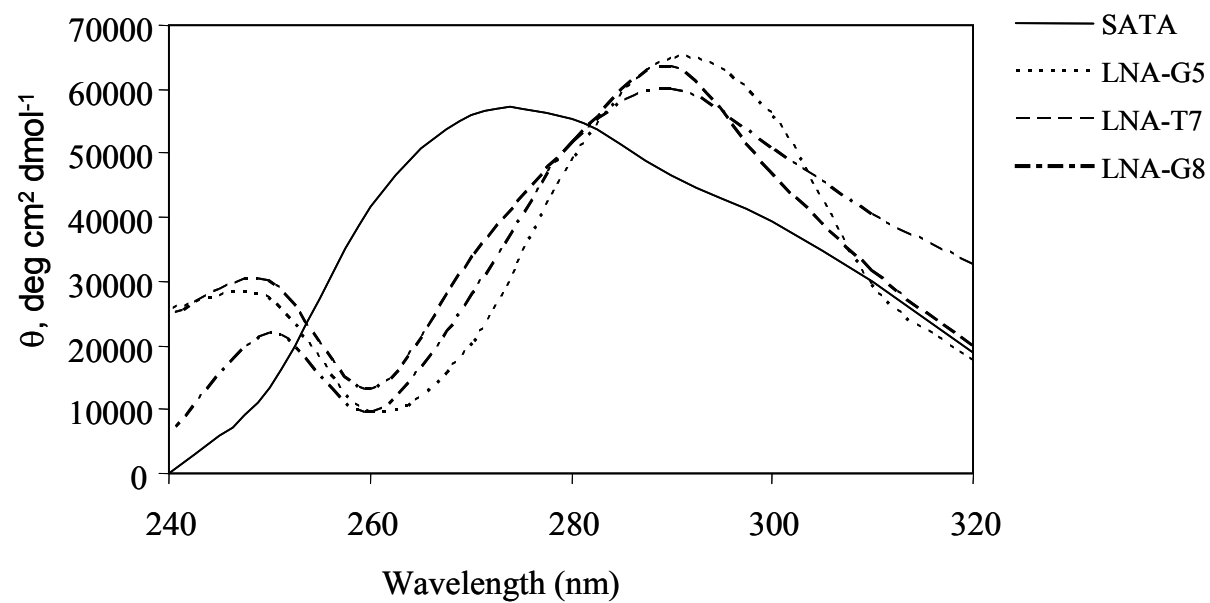


Upon heating to $95^{\circ} \mathrm{C}$, this signal disappeared suggesting a thermally labile structure as expected (data not shown). By contrast, the scrambled aptamer, SATA, displayed a spectrum consistent with single-stranded, random coil DNA with a strong positive absorbance from base stacking at $275 \mathrm{~nm}$ and a minimum at $238 \mathrm{~nm}$.

The thermal stability of the thrombin aptamer and the LNA-substituted aptamers was determined using UV melting curves in buffer containing $\mathrm{K}^{+}$. The LNA substitution had either a moderate stabilizing or destabilizing effect on the folded structure, depending on the position of the LNA in the thrombin aptamer sequence, though we were unable to measure the melting temperature for the aptamer with LNA in position T4, presumably because it is particularly unstable (Table 1). Interestingly, the melting curves of the LNA-G5 and LNA-G8 thrombin aptamers were hyperchromic instead of the expected hypochromic change (Figure 3).

Figure 3. UV melting curves for LNA-substituted and unsubstituted thrombin aptamers.
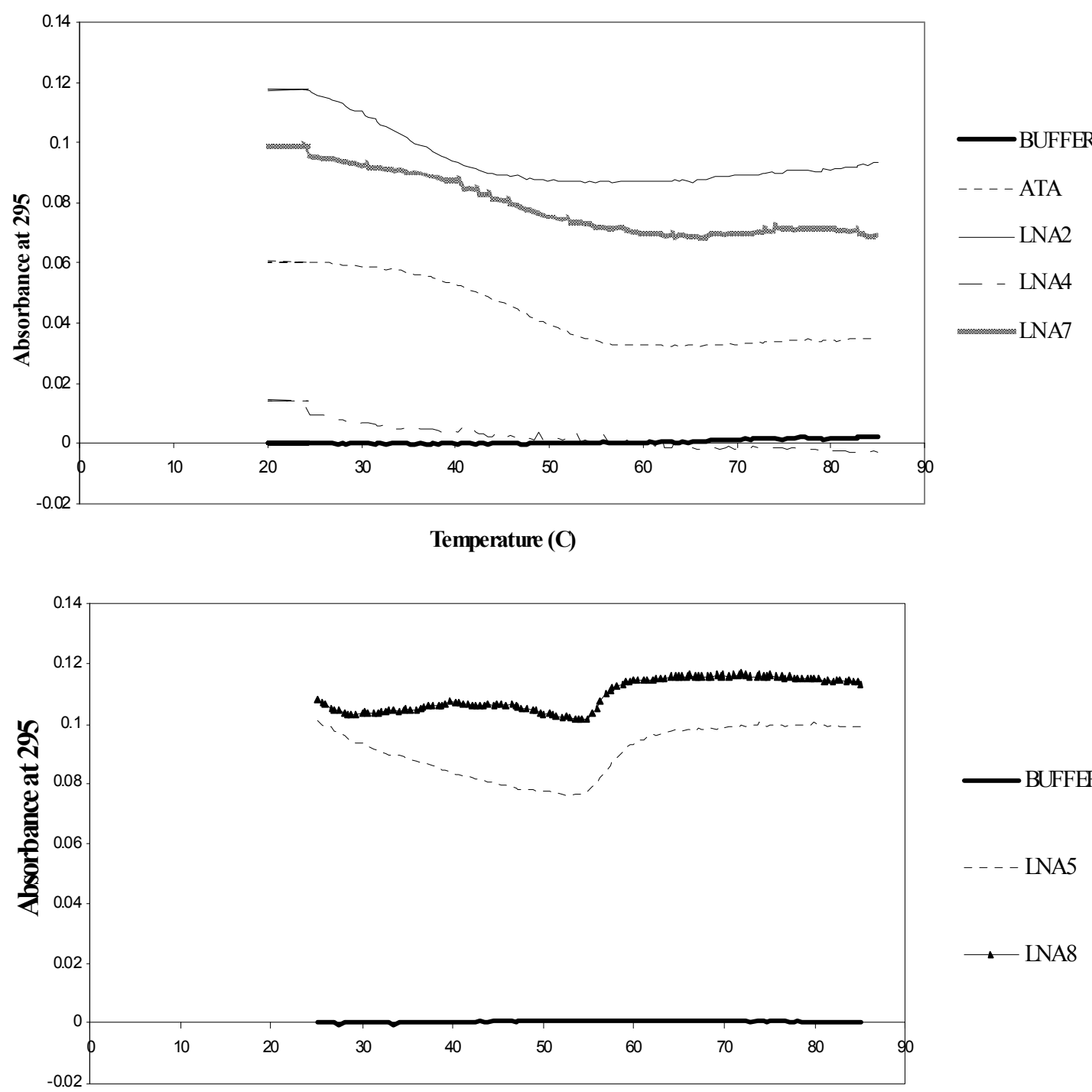
Table 1. Thermal stabilites of G-quadruplexes used in this study. (a) Thermal stability was determined by using the first derivative of the absorbance versus temperature curve (b) The first oligonuceotide is the control, un-substituted thrombin aptamer (ATA). Subsequent oligonuceotides contain LNA at the locations indicated by the listed base. (b) The melting temperature could not be determined for this aptamer.

\begin{tabular}{|c|c|c|}
\hline Name & $\mathrm{dA} / \mathrm{dT}\left({ }^{\circ} \mathrm{C}\right)^{\mathrm{a}}$ & Oligonucleotide $^{\mathrm{b}}$ \\
\hline ATA & 48.1 & $5^{\prime} d$ (GGTTGGTGTGGTTGG) \\
\hline LNA-G2 & 33.48 & $5^{\prime} d(-G-------)$ \\
\hline LNA-T4 & n.d. ${ }^{\mathrm{b}}$ & $5^{\prime} d(--T-------)$ \\
\hline LNA-G5 & 50.7 & $5^{\prime} d(---G------)$ \\
\hline LNA-T7 & 43.4 & $5^{\prime} d(----T-----)$ \\
\hline LNA-G8 & 50 & $5^{\prime} d(-----G-----)$ \\
\hline
\end{tabular}

Combined with the CD spectroscopy data, the biophysical characterization of the thrombin aptamer and LNA-containing derivatives confirmed the presence of an anti-parallel G-quadruplex in the LNAsubstituted aptamers. We also observed both stabilizing and destabilizing effects of the LNA, which is consistent with our previous report that LNA substitution demonstrates a position dependent effect on the stability of G-quadruplexes [9]. Interestingly, we did not observe a change in folding topology of the thrombin aptamer to a parallel quadruplex, which would generate a CD spectrum with a maximum at $260 \mathrm{~nm}$. This contrasts our findings with a telomeric G-quadruplex, for which LNA could induce folding into a parallel structure [9]. Perhaps the thrombin aptamer is incapable of producing a parallel G-quadruplex. One recent report shows that LNA at position 15 of the thrombin aptamer results in formation of an anti-parallel G-quadruplex, consistent with our interpretation [18]. Further analysis of the substituted aptamers by NMR will be required to determine their fine structure.

Figure 4. Thrombin inhibition by thrombin aptamers as assessed by a fibrinogen clotting assay. Inhibition of thrombin aptamers was determined by measuring the residual thrombin activity after incubation with the indicated thrombin aptamer. Data are representative of at least five data sets and the standard deviation between data sets was $<5 \%$.

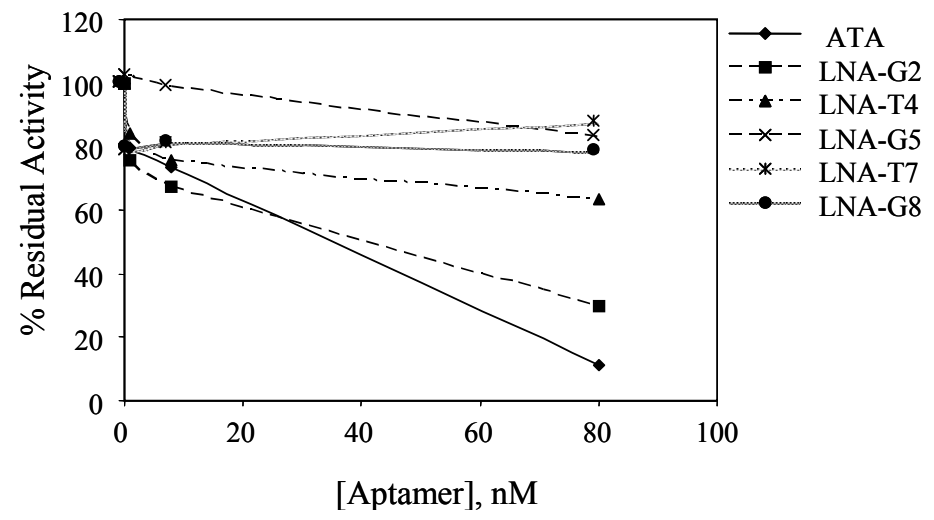




\section{Biological Activity of Thrombin Aptamers}

The effect of LNA substitution on the biological activity of the thrombin aptamer was determined using two different assays: fibrinogen clotting assays and heparin template curve assays, in which heparin cofactor II (HCII) and anti-thrombin III (ATIII) thrombin-inhibition assays are performed in the presence of heparin. Previously, the quadruplex structure of the aptamer was shown to be essential for activity, as the scrambled aptamer used during characterization steps was shown to be inactive [19]. Generally, the un-substituted thrombin aptamer exhibited a greater capacity for inhibiting fibrinogen clotting activity than did the LNA substituted derivatives (Figure 4). LNA substitutions at G5, T7, or G8 dramatically reduced fibrinogen clotting activities, whereas LNA substitutions at G2 and T4 only had slightly decreased inhibitory activity when compared to the un-substituted thrombin aptamer. These results were not expected because, in general, the stability of a biologically active Gquadruplex coincides with activity. Interestingly, the LNA-G2 thrombin aptamer was one of the least stable quadruplexes (as determined by thermal melting curves) but demonstrated activity equal to the control aptamer. By contrast, LNA-G5 and G8 thrombin aptamers were more stable than the control thrombin aptamer but were less active with respect to inhibition of fibrinogen clotting activity.

Figure 5. ATIII-heparin inhibition of thrombin in the presence of thrombin aptamers. The rate of thrombin inhibition by ATIII was determined using final concentrations of $1 \mathrm{nM}$ thrombin, $10 \mathrm{nM}$ ATIII, $5 \mathrm{nM}$ aptamer, and increasing concentrations of heparin from 0.1 to $10,000 \mu \mathrm{g} / \mathrm{ml}(6.7-66,667 \mathrm{nM})$.

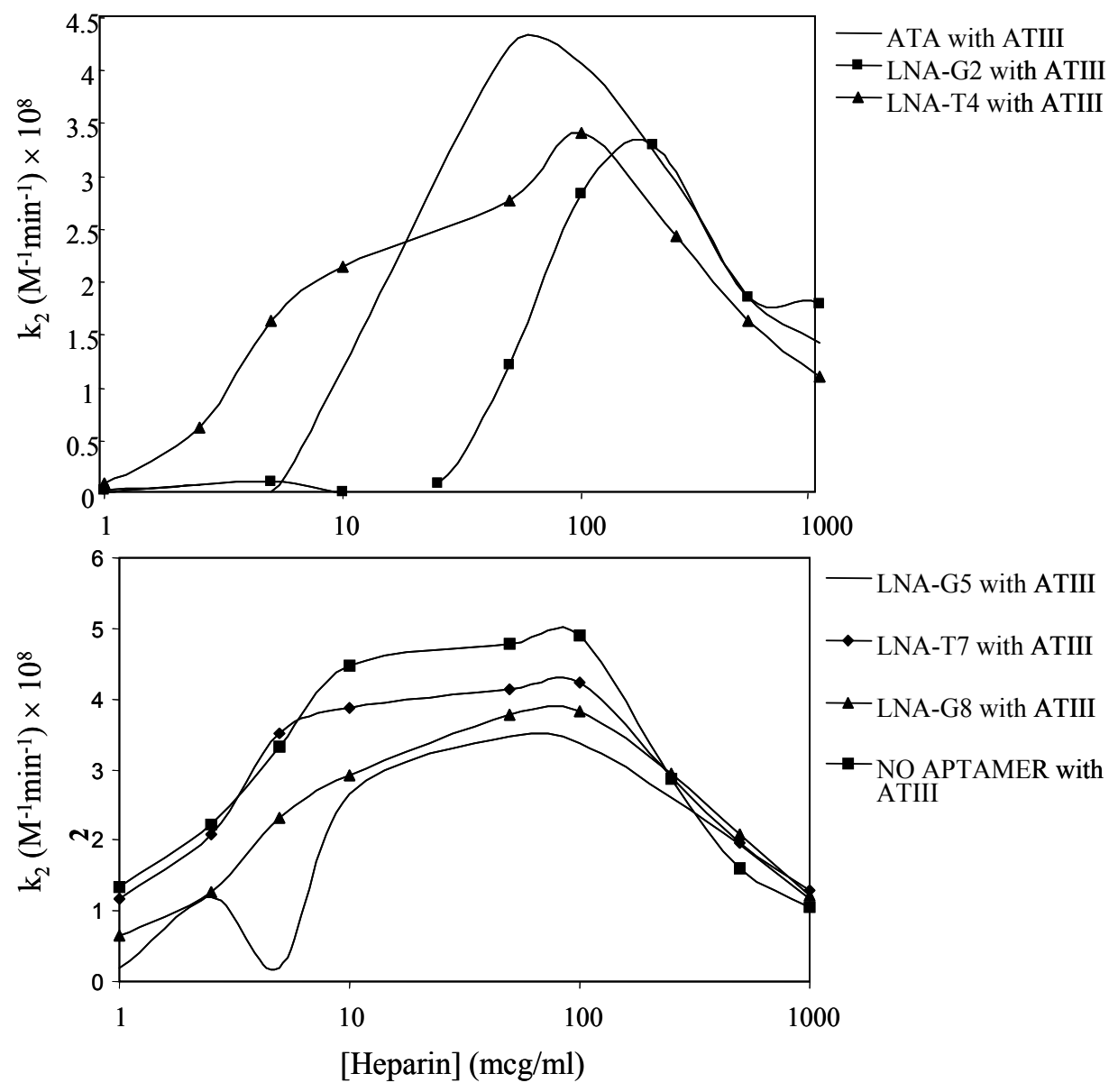


It is possible that LNA substitutions could influence the structure of the thrombin aptamer and change the nature of aptamer-thrombin interaction. To test if the LNA-substituted aptamers bound to a different site on thrombin, we utilized heparin template curve assays in the presence of serpins that are thrombin inhibitors. Two serine protease inhibitors (serpins) were used for these assays, HCII and ATIII. Both serpins were examined because HCII requires thrombin exosite-1 as part of its inhibitory mechanism, while ATIII requires thrombin exosite 2 but not exosite- 1 for its inhibition of thrombin in the presence of heparin. Thus, binding of the thrombin aptamer should interfere with binding of HCII to thrombin but not perturb the binding of ATIII. We would expect a similar effect on the rate of thrombin inhibition by each serpin if the LNA-substituted aptamers bind exosite- 1 in the same manner as the un-substituted thrombin aptamer. Consistent with this expectation, we found that the presence of the substituted and control aptamers did not influence ATIII-mediated thrombin inhibition in the presence of heparin (Figure 5).

Figure 6. HCII-heparin inhibition of thrombin in the presence of thrombin aptamers. The rate of thrombin inhibition by HCII was determined using final concentrations of $1 \mathrm{nM}$ thrombin, $10 \mathrm{nM} \mathrm{HCII,} 5 \mathrm{nM}$ aptamer, and increasing concentrations of heparin from 0.1 to $10,000 \mu \mathrm{g} / \mathrm{ml}(6.7-66,667 \mathrm{nM})$.

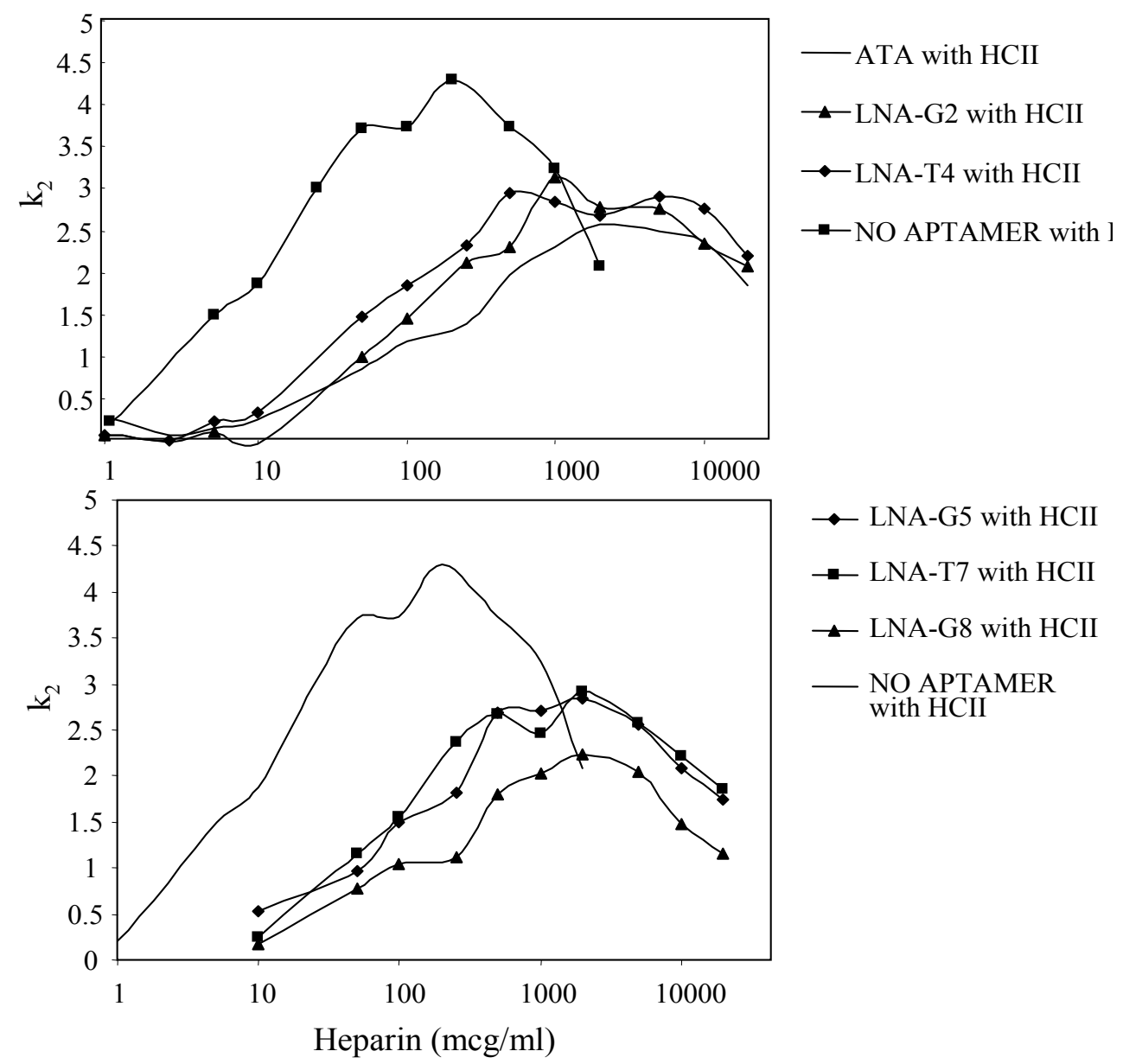

In contrast, we found that a greater concentration of heparin was required to achieve the maximal $k_{2}$ for the thrombin inhibition reaction when HCII was in the presence of either the substituted or control aptamers (Figure 6). This suggests that the LNA substituted and control thrombin aptamers both bind 
to exosite-1 and do not interfere with serpin binding to exosite 2 . We were surprised to find that the aptamers with LNA substitutions at positions G5, T7, or G8 did not inhibit fibrinogen clot formation of thrombin, but did affect HCII-mediated thrombin inhibition in the presence of heparin. Collectively, the results suggest that these LNA-containing aptamers do bind to thrombin and interact specifically with thrombin exosite-1, but they also suggest subtle changes either in binding affinities or their ability to bind unique thrombin exosite residues. The lower activity of the LNA-aptamers suggest that subtle changes in the aptamer binding to thrombin exosite- 1 contribute to anti-thrombin activities as demonstrated here with two different thrombin exosite-1-dependent reactions, fibrinogen clotting and $\mathrm{HCII} /$ heparin-catalyzed thrombin inhibition.

The disparity between LNA-induced effects on biological activity and stability of the aptamers is likely due to subtle local structural effects rather than global structural effects or effects on thermal stability. It appears that the location of the LNA substituted bases within the quadruplex structure dictates the biological activity but not necessarily thermal stability. One possibility is that the conditions used in the biological assays stabilize the modified aptamers. However, even if this were the case, it does not seem likely that the stabilizing effect would be sequence specific in a manner not reflected by the thermal stability studies. It is more likely that substituting LNA into position G5, T7, or G8 interferes directly with binding between thrombin and the aptamer. Of note, T4 is positioned in one of the two minor T-loops of the aptamer's G-quadruplex structure (Figure 1). Hydrogen bonding and base stacking interactions between T4 and T13 in the aptamer have been shown to be integral for the aptamer's ability to inhibit thrombin. For example, a T to A substitution at T4 resulted in a loss of thrombin inhibition [23]. It is therefore possible that a substitution of LNA into position 4 could serve to attenuate thrombin inhibitory activity by exerting a destabilizing effect on the aptamer. As mentioned earlier, T7 and G8 LNA-substituted aptamers both showed relatively weak inhibition of thrombin and are located in the major T-loop of the quadruplex. Perhaps the decrease in thrombin inhibition activity of LNA5, LNA7, and LNA8 is due to interference of specific protein-DNA interactions.

In summary, we have demonstrated that LNA can substitute for normal DNA in a biologically active G-quadruplex. These data can be combined with those published by Virno et al. [17], to generate a structure activity relationship for the thrombin aptamer. Virno et al. showed that substitution with LNA of all residues, all guanosine residues, or only the G1 residue resulted in inactive aptamers, whereas substitution at G15 resulted in partial activity even though the G15 substituted structure folded into a stable quadruplex. Combined with our results, we conclude that for the thrombin aptamer, overall G-quadruplex stability is not the major contributor to biological activity, which is unexpected. Instead, the local structure of the TGT loop appears to dominate activity, presumably due to its direct interaction with thrombin at exosite-1. 


\section{Experimental Procedures}

\section{Materials}

Oligonucleotides

The thrombin aptamer d(GGTTGGTGTGGTTGG) was obtained from Integrated DNA Technologies (Coralville, IA). Thrombin aptamer analogs containing individual LNA substitutions in positions G2, T4, G5, T7, or G8 were provided by Proligo (Boulder, CO). All aptamers were purified by denaturing polyacrylamide gel electrophoresis. Gels were prepared with $20 \%$ acrylamide, $7 \mathrm{M}$ urea, and $1 \times$ TBE ( $89 \mathrm{mM}$ Tris, $89 \mathrm{mM}$ boric acid, $2 \mathrm{mM}$ EDTA, $\mathrm{pH} 8.5$ ) and $1 \times$ TBE was used as the running buffer. Oligonucleotide bands were visualized by UV shadowing and extracted from the gel by the crush and soak method. Briefly, gel slices were crushed with a glass rod and extracted twice with TEN (10 mm Tris-HCl, pH 8.0, $1 \mathrm{~mm}$ EDTA, and $200 \mathrm{mM} \mathrm{NaCl}$ ). Purified aptamers were concentrated by ethanol precipitation and resuspended in TE $(10 \mathrm{~mm}$ Tris-HCl, $\mathrm{pH} 8.0$, and1 mm EDTA). Aptamer concentrations were determined by UV spectroscopy using an extinction coefficient of $143,300 \mathrm{M}^{-1} \mathrm{~cm}^{-1}$ and were determined to be single sequences by PAGE analysis.

General Materials

HNPK (20 mM HEPES, $145 \mathrm{mM} \mathrm{NaCl}, 5 \mathrm{mM} \mathrm{KCl}, 0.1 \%$ polyethylene glycol 8000, $0.02 \% \mathrm{NaN}_{3}$ at $\mathrm{pH}$ 7.4) was used as the buffer in all coagulation studies. TEK (10 mM Tris pH 7.5, $1 \mathrm{mM}$ EDTA and $50 \mathrm{mM} \mathrm{KCl}$ ) was used as the buffer for thermal stability analyses and $\mathrm{CD}$ experiments. Thrombin solutions were prepared from a stock solution of human $\alpha$-thrombin that was purified as described previously [19].

\section{Methods}

Fibrin Clotting Assays

Continuous kinetic assays were performed in standard U-bottom, ovalbumin (OVA)-coated, 96 well microtiter plates at room temperature. Solutions of thrombin, fibrinogen, and aptamer were prepared in HNPK. In individual wells, thrombin $(2 \mathrm{nM})$ was incubated with increasing amounts of aptamer (1 $\mathrm{nM}, 10 \mathrm{nM}, 100 \mathrm{nM})$ for $1 \mathrm{~min}$. After $1 \mathrm{~min}$, fibrinogen $(4 \mathrm{mg} / \mathrm{ml})$ was added and clotting was measured by UV absorbance and reported as residual thrombin activity [20]. Each reaction was performed in duplicate and negative controls included duplicate samples containing no aptamer, and reactions containing a scrambled aptamer d(GGTGGTGGTTGTGGT).

Heparin Template Curves

HNPK was used as the buffer for these assays which were performed with $1 \mathrm{nM}$ thrombin, $10 \mathrm{nM}$ serpin [heparin cofactor II (HCII) or anti-thrombin III (ATIII)], $5 \mathrm{nM}$ thrombin aptamer, 150 $\mu \mathrm{M}$ tosyl-Gly-Pro-Arg-p-nitroanilide (GPA), and increasing concentrations of heparin (0.6-20,000 $\mu \mathrm{g} / \mathrm{ml}$ ). Thrombin was added 15 seconds before the addition of the substrate GPA, and the reaction was quenched $10 \mathrm{~min}$ later by the addition of $50 \mu \mathrm{L}$ of $50 \%$ acetic acid. Reactions with each 
concentration of heparin were performed in quadruplet and corresponding controls containing no HCII or ATIII were performed in duplicate. After reactions were quenched, plates were covered with microplate adhesive film and spun at $2800 \mathrm{rpm}$ for $15 \mathrm{~min}$ at $\mathrm{rt} .100 \mu \mathrm{L}$ of each supernatant was removed and analyzed by UV at $405 \mathrm{~nm}$ using a Thermomax microtiter plate reader (Molecular Devices). Rate constants were calculated as described by Ciaccia et al [21] and are reported as secondorder rate constants of inhibition $\left(\mathrm{M}^{-1} \mathrm{~min}^{-1}\right)$ and were plotted vs heparin concentration.

Thermal Stability Analysis

Thermal stability of the thrombin aptamer and LNA-substituted aptamers was determined by UV melting curves obtained on a Perkin Elmer UV/Vis Spectrophotometer Lambda 20 using a PTP-6 Peltier System to control the temperature. Aptamers were prepared as $5 \mu \mathrm{M}$ samples in $1 \mathrm{X}$ TEK in a final volume of $400 \mu \mathrm{L}$. Samples were heated from $20-85^{\circ} \mathrm{C}$, at $1{ }^{\circ} \mathrm{C} \mathrm{min}^{-1}$, and the absorbance at 295 $\mathrm{nm}$ was recorded [22]. Absorbance was measured for both melting and reannealing of the aptamers. Melting temperatures were calculated from the first derivative of the duplicate absorbance versus temperature curves.

Circular Dichroism

Oligonucleotides $(5 \mu \mathrm{M})$ were annealed by heating samples for 5 min at $95^{\circ} \mathrm{C}$ then slow cooling $\left(1^{\circ} \mathrm{C} \mathrm{min}^{-1}\right)$ to room temperature in $400 \mu \mathrm{l} 1 \mathrm{X}$ TEK buffer. CD spectra were recorded on an Applied Photophysics Pistar-180 Circular Dichroism spectrophotometer equipped with a Peltier temperature controller. For each sample, spectral scans were collected over the wavelength range 200-350 nm at $25^{\circ} \mathrm{C}$ in a $1 \mathrm{~mm}$ path length cell at a scanning rate of $20 \mathrm{~nm} \mathrm{~min}^{-1}$. Separate spectra were obtained at $95^{\circ} \mathrm{C}$. Three scans were averaged for each sample, and the scan of the buffer alone was subtracted for each sample. CD spectra were collected in units of millidegrees versus wavelength and normalized to the strand concentration and number of residues.

\section{Acknowledgements}

This work was supported by a grant from the North Carolina Pharmacy Foundation and in part by Research Grant HL-32656 and HL-06350 (to F.C.C) from the National Institutes of Health. M.B.J. was supported in part by a R.J. Reynolds Fund Award from the University of North Carolina and a New Investigator Award from the American Association of Colleges of Pharmacy. The University of North Carolina at Chapel Hill's Office of the Vice Chancellor for Research and Economic Development provided support for open access publication.

\section{References and Notes}

1. Hotoda, H.; Koizumi, M.; Koga, R.; Kaneko, M.; Momota, K.; Ohmine, T.; Furukawa, H.; Agatsuma, T.; Nishigaki, T.; Sone, J.; Tsutsumi, S.; Kosaka, T.; Abe, K.; Kimura, S.; Shimada, K. Biologically active oligodeoxyribonucleotides. 5. 5'-End-substituted d(TGGGAG) possesses anti- 
human immunodeficiency virus type 1 activity by forming a G-quadruplex structure $J$. Med. Chem. 1998, 41, 3655-3663.

2. Dapic, V.; Bates, P.J.; Trent, J.O.; Rodger, A.; Thomas, S.D.; Miller, D.M. Antiproliferative activity of G-quartet-forming oligonucleotides with backbone and sugar modifications. Biochemistry 2002, 41, 3676-3785.

3. Griffin, L.C.; Tidmarsh, G.F.; Bock, L.C.; Toole, J.J.; Leung, L.L.. In vivo anticoagulant properties of a novel nucleotide-based thrombin inhibitor and demonstration of regional anticoagulation in extracorporeal circuits. Blood 1993, 81, 3271-3276.

4. Macaya, R.F.; Schultze, P.; Smith, F.W.; Roe, J.A.; Feigon, J. Thrombin-binding DNA aptamer forms a unimolecular quadruplex structure in solution. Proc. Natl. Acad. Sci. USA 1993, 90, $3745-$ 3749.

5. Padmanabhan, K.; Padmanabhan, K.P.; Ferrara, J.D.; Sadler, J.E.; Tulinsky, A. The structure of alpha-thrombin inhibited by a 15-mer single-stranded DNA aptamer. J. Biol. Chem. 1993, 268, 17651-17654.

6. Li, W.X.; Kaplan, A.V.; Grant, G.W.; Toole, J.J.; Leung, L.L. A novel nucleotide-based thrombin inhibitor inhibits clot-bound thrombin and reduces arterial platelet thrombus formation. Blood 1994, 83, 677-682.

7. Keniry, M.A. Quadruplex structures in nucleic acids. Biopolymers 2000, 56, 123-146.

8. Kelly, J.A.; Feigon, J.; Yeates, T.O. Reconciliation of the X-ray and NMR structures of the thrombin-binding aptamer d(GGTTGGTGTGGTTGG). J. Mol. Biol. 1996, 256, 417-422.

9. Dominick, P.K.; Jarstfer, M.B. A conformationally constrained nucleotide analogue controls the folding topology of a DNA g-quadruplex. J. Am. Chem. Soc. 2004, 126, 5050-5051.

10. Petersen, M.; Wengel, J. LNA: a versatile tool for therapeutics and genomics. Trends Biotechnol. 2003, 21, 74-81.

11. Kaur, H.; Babu, B.R.; Maiti, S. Perspectives on chemistry and therapeutic applications of Locked Nucleic Acid (LNA). Chem. Rev. 2007, 107, 4672-4697.

12. Dash, C.; Yi-Brunozzi, H.Y.; Le Grice, S.F. Two modes of HIV-1 polypurine tract cleavage are affected by introducing locked nucleic acid analogs into the (-) DNA template. J. Biol. Chem. 2004, 279, 37095-37102 .

13. Johnson, M.P.; Haupt, L.M.; Griffiths, L.R. Locked nucleic acid (LNA) single nucleotide polymorphism (SNP) genotype analysis and validation using real-time PCR. Nucleic Acids Res. 2004, 32, e55.

14. Schubert, S.; Furste, J.P.; Werk, D.; Grunert, H.P.; Zeichhardt, H.; Erdmann, V.A.; Kurreck, J. Gaining target access for deoxyribozymes. J. Mol. Biol. 2004, 339, 355-363.

15. Braasch, D.A.; Corey, D.R. Locked nucleic acid (LNA): fine-tuning the recognition of DNA and RNA. Chem. Biol. 2001, 8, 1-7.

16. Simonsson, T. G-quadruplex DNA structures--variations on a theme. Biol. Chem. 2001, 382, 621628.

17. Randazzo, A.; Esposito, V.; Ohlenschlager, O.; Ramachandran, R.; Virgilio, A.; Mayol, L. Structural studies on LNA quadruplexes. Nucleosides Nucleotides Nucleic Acids 2005, 24, 795800 . 
18. Virno, A.; Randazzo, A.; Giancola, C.; Bucci, M.; Cirino, G.; Mayol, L. A novel thrombin binding aptamer containing a G-LNA residue. Bioorg. Med. Chem. 2007, 15, 5710-5718.

19. Griffith, M.J.; Noyes, C.M.; Church, C.F. Reactive site peptide structural similarity between heparin cofactor II and antithrombin III. J. Biol. Chem. 1985, 260, 2218-2225.

20. Holland, C.A.; Henry, A.T.; Whinna, H.C.; Church, F.C. Effect of oligodeoxynucleotide thrombin aptamer on thrombin inhibition by heparin cofactor II and antithrombin. FEBS Lett. 2000, 484, 87-91.

21. Ciaccia, A.V.; Monroe, D.M.; Church, F.C. Arginine 200 of heparin cofactor II promotes intramolecular interactions of the acidic domain. Implication for thrombin inhibition. J. Biol. Chem. 1997, 272, 14074-14079.

22. Mergny, J.L.; Phan, A.T.; Lacroix, L. Following G-quartet formation by UV-spectroscopy. FEBS Lett. 1998, 435, 74-78.

23. Tsiang, M.; Gibbs, C.S.; Griffin, L.C.; Dunn, K.E.; Leung, L.L. Selection of a suppressor mutation that restores affinity of an oligonucleotide inhibitor for thrombin using in vitro genetics. J. Biol. Chem. 1995, 270, 19370-19736.

(C) 2008 by MDPI (http://www.mdpi.org). Reproduction for noncommercial purposes permitted. 